\title{
Ensuring the integrity of research in the Age of open access. A proposal : hashing + public-key cryptography signing.
}

\author{
Camille Akmut
}

\begin{abstract}
On ensuring the integrity of research documents in the digital age, a concern of prime importance to the development of open access. New and old solutions are presented, and a utility tool (tenaciouscookie).
\end{abstract}




\section{Introduction}

In this report, we propose that where research goes, a way to ensure their integrity is given by the formula : hashing + public-key cryptography signing.

We look at the "old way" of how this would be done, and detail the steps.

We look at a new way of doing this with a utility called tenaciouscookie - named so in homage to Aaron Swartz who worked in all of these areas.

In general, such utilities ought to be developed to make the life of researchers easier. And, their development expended to and included in the Web, in various forms (e.g. open access platforms, open access journals, etc., submissions (forms)).

\section{Hashing, Public-key signing. The "old way"}

We go through the steps of hashing and public-key cryptography signing by using a real example.

\subsection{Hash functions}

Given a document document.pdf (or any other document) produced by a researcher : we detail the steps of hashing followed by public-key signing, the method we propose ought to be used by researchers to digitally ensure the integrity of their research (and towards their readers).

To facilitate narration, discussion and reasoning, we choose a real document produced by a researcher ${ }^{1}$.

\footnotetext{
${ }^{1}$ Yitang Zhang. "On the Landau-Siegel Zeros Conjecture". https://arxiv.org/abs/ 0705.4306. Submitted on 29 May 2007.
} 


\title{
On the Landau-Siegel Zeros Conjecture
}

\author{
Table of Content \\ Yitang Zhang \\ 1. Introduction \\ 2. The Set $\Psi^{*}$ \\ 3. Zeros of $L(s, v) L(s, v x)$ in $n$ \\ 4. The Functions $\mathcal{K}_{ \pm}(s, \psi)$ \\ 5. The Linear Funetional $\oplus(f ; \rho, \varphi)$ \\ 6. Solutions to Boundary Value Problems \\ 7. Approximation to po \\ 8. The Fundamental Inequality: Preliminary \\ 9. Upper Bounds for $\Theta(k)$ and $\Theta(r)$ \\ 10. Asymptotic Expression for $\Theta\left(\varphi_{0}\right)$ \\ 11. The Fundamental Inequality: Completion \\ 12. Est imation of $\mathcal{E}$ \\ 13. Proof of Theorem 1 \\ Reference:
}

\section{Introduction}

The main result of this paper is the following

Theorem 1 For ang real primitive character $x$ of modalus $D$ we have

$$
L(1, x)>c_{1}(\log D)^{-17}(\log \log D)^{-1}
$$

ahere $\mathrm{Q}>0$ is an effecticely compatable onstant.

As a direct conscquence of Theorem 1 , we have

Theorem 2 Let $\mathrm{X}(\bmod D)$ be as in Therem 1. Then ave have

$$
L(\sigma, x) \neq 0
$$


This hypothetical researcher would proceed to produce what is called a "hash" (a digital signature) for (their) this file.

Hash functions, including SHA-1, are described in Chap. 11. For now it is sufficient to know that SHA-1 compresses $\mathrm{x}$ and computes a 160-bit fingerprint. This fingerprint can be thought of as a representative of $\mathrm{x} .^{2}$

When we talk of hashing, hashes, or hash values (more) precisely, we use terminology that is important to keep in mind : additional terms such as "digest" are often found in discussions relating to their uses, and it is good to have a correct idea of their precise meanings.

Hash functions are an important cryptographic primitive and are widely used in protocols. They compute a digest of a message which is a short, fixed-length bit- string. For a particular message, the message digest, or hash value, can be seen as the fingerprint of a message, i.e., a unique representation of a message. ${ }^{3}$

There are thus the message - that which must be encoded - and the message digest - the resulting hash value.

We demonstrate this on the basis of a primitive example.

Apply the hash function, SHA-1, to the message "hello";

And, give back the message digest.

a af 4 c $61 d d c c 5 e 8 a 2 d a b e d e 0 f 3 b 482$ cd 9 a ea $9434 d$

And, in real software (here using the Python Standard Library built-in hashlib module of various hash functions),

>> hashlib.sha1 (b"hello").hexdigest ()

' 6 f 9 b 9 af 3 cd 6 e 8 b8a73c2cdced 37 f e 9 f 59226 e 27 d '

Or,

\footnotetext{
${ }^{2}$ Paar and Pelzl 2009. Chapter 10, 10.4.
}

${ }^{3}$ Ibid. Chapter 11. 


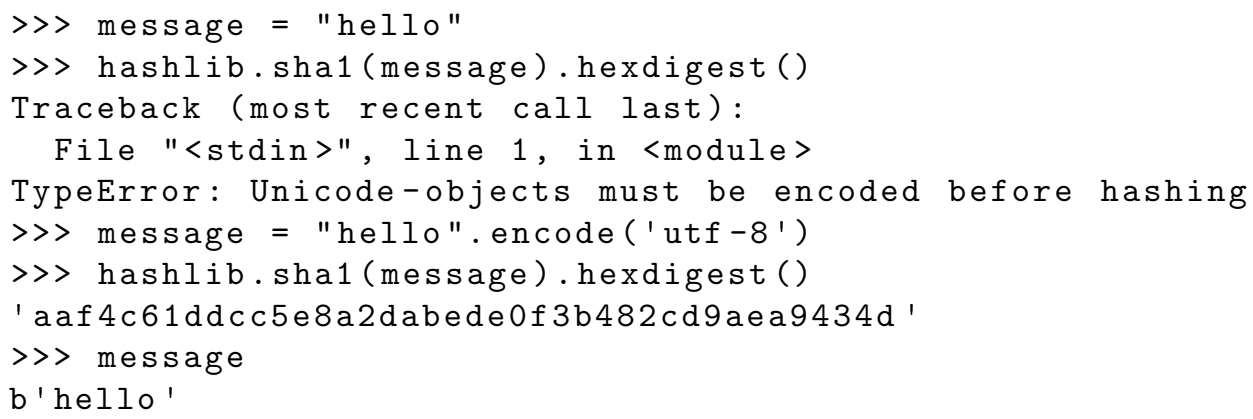

On Linux systems this is done with the series of sha<variant>sum utilities e.g. sha256sum or sha512sum, as demonstrated below :

\$ sha256sum zhang2007landausiegl.pdf

c 988 b 4 a 4 e $2036146 a$ ab 0021 fd 78 e 15 c 215 de 954170 ce $3 a$ cbfe 8430 e 9181 eb zhang 20071 andausiegl.pdf

This hash is unique to this file - "almost". Almost, probably : maybe. ${ }^{4}$

Theoretically, since the domain of SHA-256 contains $2^{2^{64-1}}$ different messages and the value set only contains $2^{256}$ different message digests, there must exist at least one possible output that has more than one possible pre-image.

Another important point is that SHA-256 is a deterministic function. This means that if you hash the same message twice, you will get the same digest both times. Hence, "almost unique" should be understood as meaning that SHA-256 will likely produce a different hash for each different input message. This might be false in an abstract mathematical sense, but it is probably true in a more practical sense:

In practice, uniqueness is not determined by the abstract theoretical non-existence of collisions, but by the practical non-existence of collisions. In order to find a collision in SHA-256, you would

\footnotetext{
${ }^{4}$ The issue is that of collision : when two different messages (can be made to) produce the same hash. Such collisions are evoked, including actual attacks of older versions of SHA, in Paar and Pelzl 2009.
} 
probably have to execute the algorithm some $2^{128}$ times. It is unlikely that this will happen anytime soon, even if you count the total number of times SHA-256 will ever be executed by anyone in the entire universe combined.

Does a message "exist" if it belongs to a well defined abstract set, or does it "exist" because it has actually been produced and has been represented by someone or something in the physical reality ${ }^{5}$

The precise uniqueness of this digital signature is related to the two formula given below :

$$
\begin{aligned}
& 2^{2^{64-1}} \\
& =2^{2^{63}} \\
& =10^{10^{18.4434 . .}} \\
& =1 \cdot 10^{184.434 . .}
\end{aligned}
$$

Which describes the domain, or set, of possible different messages. (That can be passed to the SHA-256 algorithm/function, or any such algorith$\mathrm{m}$ /function.) We understand this to be an (almost) infinite amount, and understand henceforth $2^{2^{64-1}}$ to be some approximation of (almost-)infinity.

$$
\begin{aligned}
& 2^{256} \\
& =1.1579 . . \cdot 10^{77}
\end{aligned}
$$

Which describes the domain, or set, of possible different values ("signatures") that can be generated by (with) the SHA-256 algorithm. This is a finite set, we understand.

\section{$1.2 \quad$ Public-private key signing}

A researcher would then go on to create a pair of public-private keys.

This is done according to the GNU Privacy Guard (GnuPG, or gpg) "The GNU Privacy Handbook" like so :

\footnotetext{
${ }^{5}$ Hellström 2015.
} 
alice $\%$ gpg --gen-key

With the following output, when done in actuality :

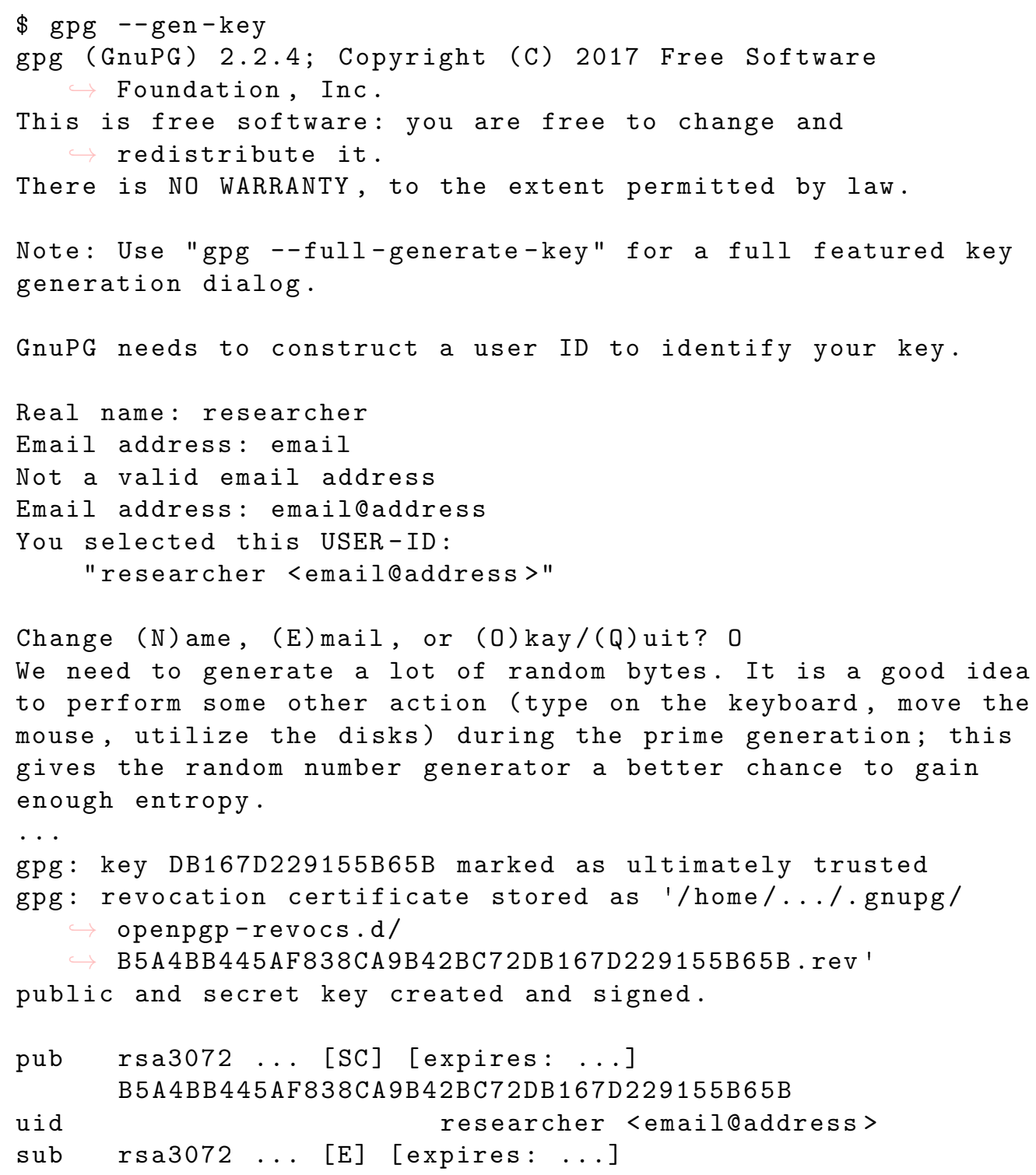

Whereas the alternative command appearing as a note gives the following 


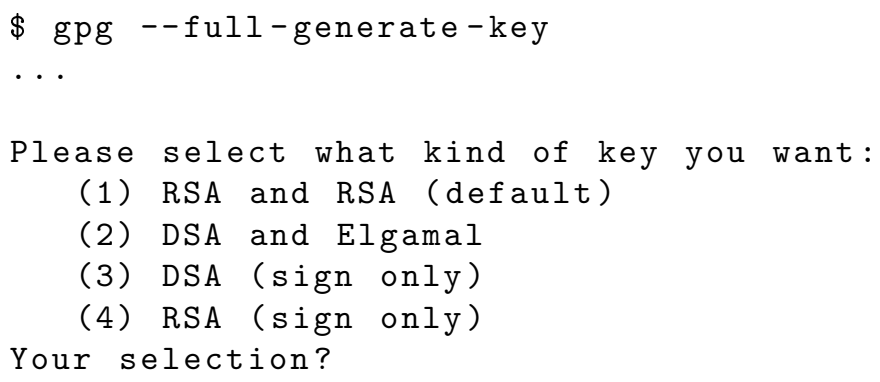

A user (researcher) may then check for (the existence of) their key :

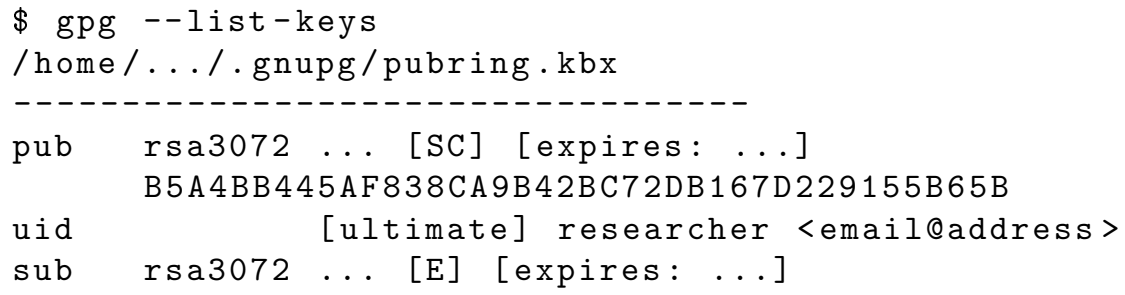

By which command, gpg --list-keys, only the public key will be listed. And, for their private ("secret") key :

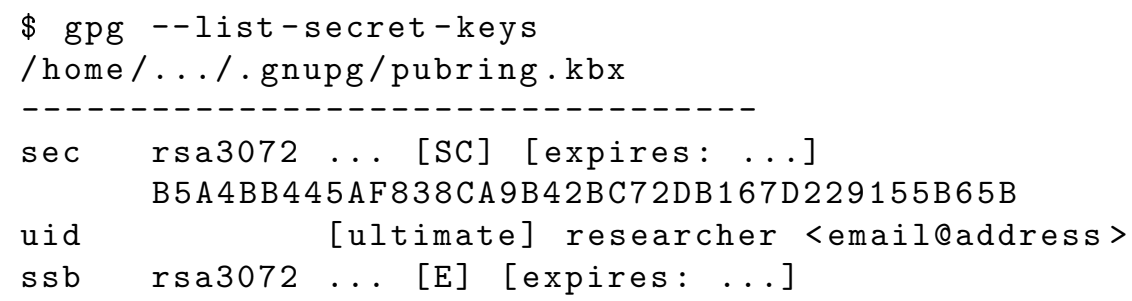

And, for both key's ID's ${ }^{6}$ :

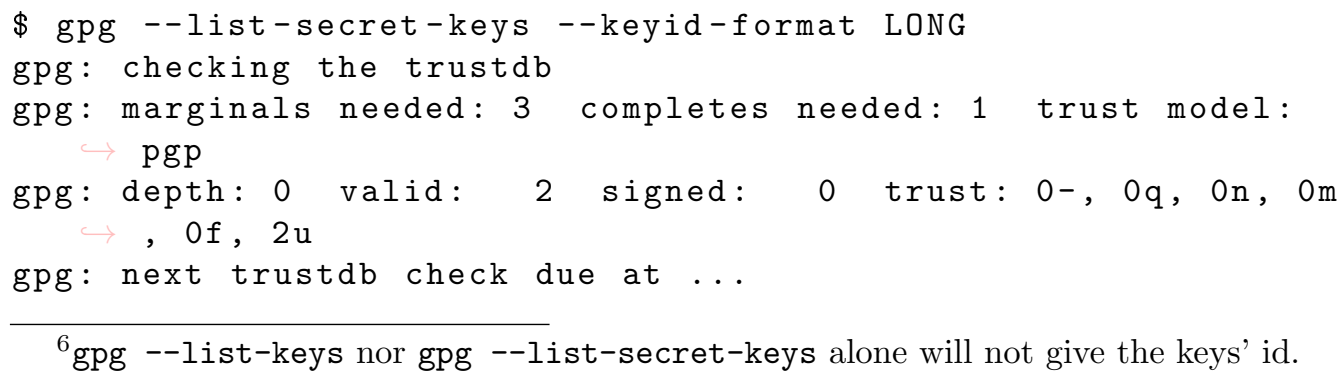




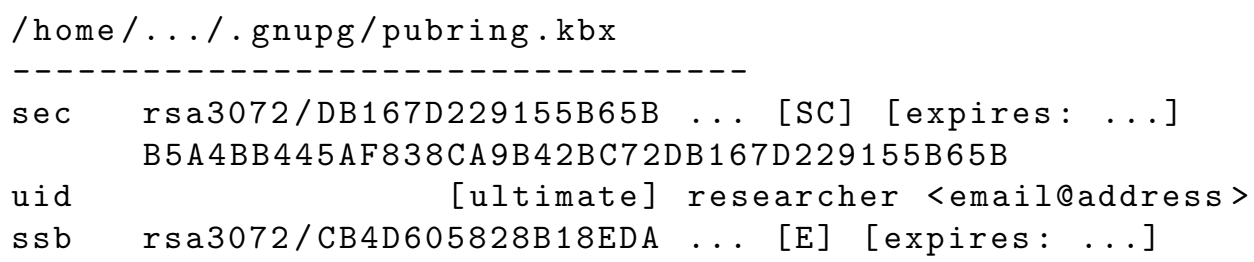

Where DB167D229155B65B describes the public key, and CB4D605828B18EDA the private key.

The key ID's are important for operations such as deletion of keys.

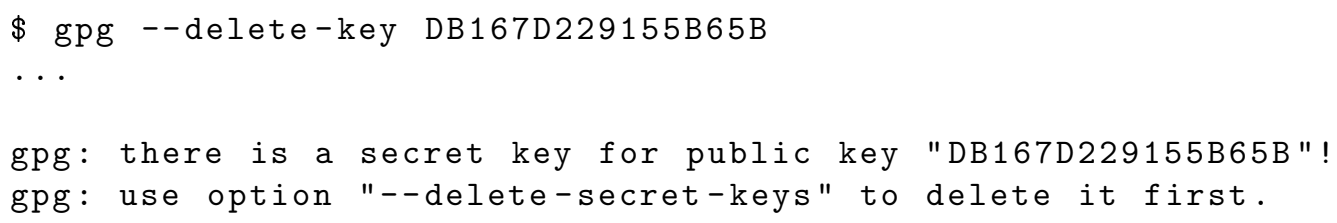

(Finally,) Signing of a file is done like so :

The command-line option -sign is used to make a digital signature. The document to sign is input, and the signed document is output.

alice\% gpg --output doc.sig--sign doc

7

And, thus :

\$ gpg --output zhang2007landausiegl.pdf.sig --sign zhang 2007 landausiegl .pdf

The output is a document zhang2007landausiegl.pdf.sig of type "detached OpenPGP signature".

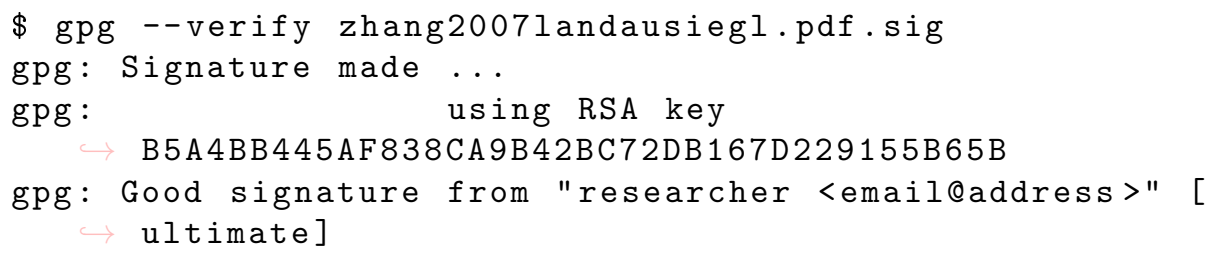

${ }^{7}$ The GNU Privacy Handbook. 
We suggest the researcher uploads this - now encrypted and signed - file to any platform - it should not matter, along with the unencrypted, unsigned file for convenience. For convenience, one version is uploaded encrypted, one version is uploaded in the clear. But, should still be checked every time / in any case.

Given a signed document, you can either check the signature or check the signature and recover the original document. To check the signature use the -verify option. To verify the signature and extract the document use the -decrypt option. The signed document to verify and recover is input and the recovered document is output.

blake\% gpg --output doc --decrypt doc.sig

8

The signing of the original document itself through public-private key cryptography should be enough, but the hash value may be certified in the same way as well.

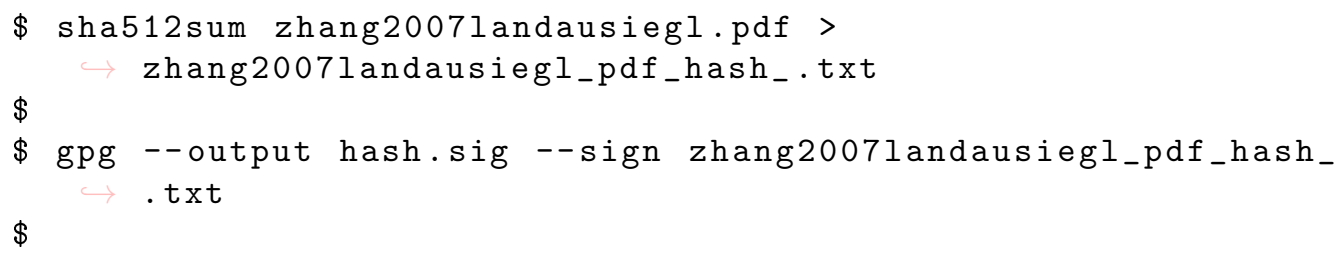

\section{2 tenaciouscookie : a utility to certify the authenticity of digitally published research.}

\section{1 sha3-tc.py : SHA3 class hash functions utility pro- gram.}

A utility called sha3-tc.py is first presented that uses the latest class of SHA3 hashing algorithms (instead of SHA2 class algorithms like SHA-256 or SHA-512) :

\section{${ }^{8}$ Ibid.}


\$ python3 sha3-tc.py zhang2007landausiegl.pdf

a 36 a abf 486471783448 f 04 b 1 b 3b 4 d 2 e eecf ca 5 c 5363 b $4169570398 d b f 35725$ fdf 3247 f $38 d a 2$

ec 4 af 8 a 13 f 38b558d073cfc 94 a 1 e 3 ed 07 ab 5551 b 97821 ed 77 e 043

Here, specifically the sha3_512 implementation of the Python Standard Library is used (the highest available to our knowledge within that same library).

It belongs to the implementations officialy recognized by the SHA3 working group. ${ }^{9}$

A sha3sum utility, of the likes of the sha<variant>sum utilities, does not currently exist for Linux.

\section{2 tenaciouscookie : easy signature creation.}

\$ python3 tc3.py zhang2007landausiegl.pdf

zhang2007landausiegl.pdf.sig signature file created for

zhang2007landausiegl.pdf

In the future, we would like to implement this as a (native) command-line utility, that may be called like so :

\$ tenaciouscookie filename.pdf

filename.pdf.sig signature file created for filename.pdf

But, for now, this is enough. Others may improve on it.

Future implementations may do hashing and signing together (in what order, or how exactly, remains an open question).

Note that this utility currently requires version 3.6 or higher or Python $(3)^{10}$.

\footnotetext{
9 "Implementations". https://keccak.team/software.html

10 "New in version 3.6: SHA3 (Keccak) and SHAKE constructors sha3_224(), sha3_256(), sha3_384(), sha3_512(), shake_128(), shake_256().”. https://docs.python.org/3/ library/hashlib.html
} 


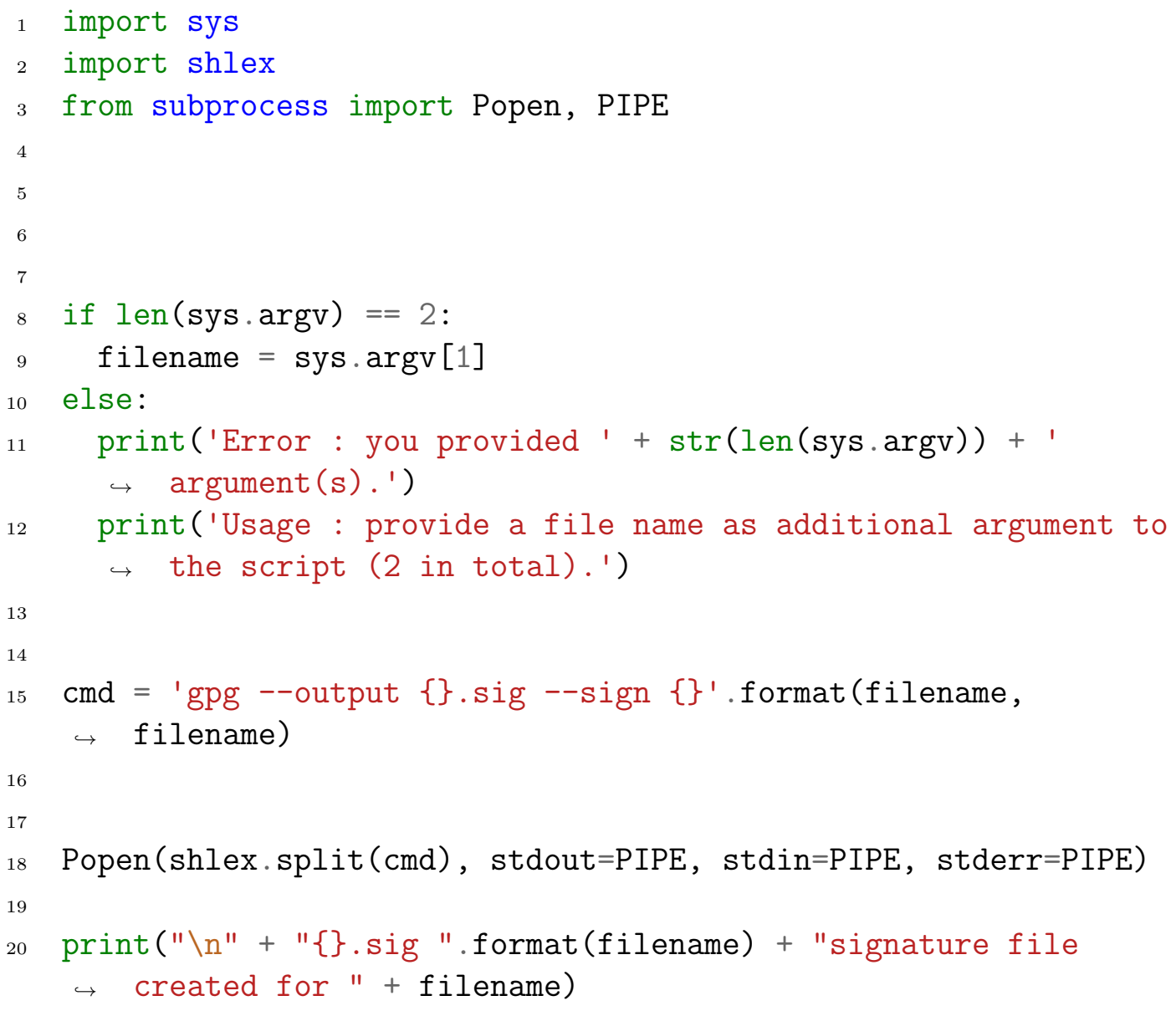




\section{Bibliography}

Paar, Christof and Pelzl, Jan. 2009. Understanding Cryptography. Springer. (In particular chapter 11 "Hash Functions".)

Python. The Python Standard Library. "hashlib - Secure hashes and message digests". https://docs.python.org/3/library/hashlib.html

Hellström, Henrick. 2015. "SHA-256 "almost unique"?". https://crypto. stackexchange. com/questions/29650/sha-256-almost-unique

The GNU Privacy Handbook. https://www.gnupg.org/gph/en/manual/ book1.html. In particular "Chapter 1. Getting Started". https://www. gnupg.org/gph/en/manual/c14.html 